\title{
JNFORIJIACJÓN
}

\section{El olivar tradicional: alternativas estratégicas de competitividad}

\author{
Por Juan Vilar Hernández,, ${ }^{* 1}$ María del Mar Velasco Gámez, ${ }^{*}$ Raquel Puentes Poyatos* \\ y Ana María Martínez Rodriguez**
}

\author{
*Departamento de Organización de Empresas, Marketing y Sociología. Universidad de Jaén. \\ Campus de Las Lagunillas s/n, 23071 Jaén-España. \\ ** Departamento de Estadística e Investigación Operativa. Universidad de Jaén. \\ Campus de Las Lagunillas s/n, 23071 Jaén-España \\ ${ }^{1}$ GEA Westfalia Separator Ibérica, S.A., Centro de Desarrollo y Competencia para Aceite de Oliva. \\ P.I. Los Cerros. Cerámica, naves 4, 5 y 6, 23400 Úbeda - España \\ (Autor para correspondencia: Juan.Vilar@geagroup.com)
}

\section{RESUMEN}

\author{
El olivar tradicional: Alternativas estratégicas de \\ competitividad
}

En los últimos años la situación competitiva de los sistemas de explotación de olivar, denominados "tradicionales" se está viendo mermada por tratarse de un modo de cultivo que adolece de una excesiva atomización, dispersión y elevados costes de explotación. El objetivo de este trabajo es corroborar, mediante un estudio empírico, si la aplicación de las estrategias de modernización y cooperación que se proponen (la modernización de la explotación de olivar tradicional, la explotación compartida, y por último mediante el sistema de cultivo asistido) mejora significativamente la competitividad del olivar tradicional. Para alcanzar el objetivo propuesto, se analizaron datos obtenidos a través de la complementación de cuestionarios a una muestra de 40 explotaciones olivareras tradicionales, 10 por cada una de las alternativas estratégicas formuladas. El análisis estadístico de los datos permitió conocer la existencia de diferencias significativas entre la renta neta de los olivicultores que desarrollan su actividad de forma tradicional frente al resto de explotaciones que adoptaban las estrategias propuestas. Los resultados del estudio muestran que la implantación de las alternativas estratégicas planteadas tiene como resultado final el incremento de la competitividad del olivar tradicional a través de la renta neta del olivicultor.

PALABRAS-CLAVE: Aceite de oliva - Competitividad Estrategias - Olivar tradicional - Rentabilidad - Sistemas de explotación del olivar.

\section{SUMMARY}

Traditional olive growing system: Strategic alternatives to improve competitiveness.

In recent years, the competitive situation of the so-called "traditional" cultivation systems has decreased because this is a cultivation system that suffers excessive fragmentation, dispersal and high operation costs. The aim of this work is to determine, through an empirical study, if the use of the modernization and cooperation strategies proposed (such as incremental innovation of traditional olive cultivation system, "shared" cultivation, and finally, the assisted cultivation system) would meaningfully improve the competitiveness of the traditional olive cultivation system. To achieve this objective, data obtained from the filling in of questionnaires has been used and 40 traditional olive farms have been analyzed, 10 farms for each of the strategic alternatives proposed. The statistical analysis of the data obtained allowed us to know the existence of meaningful differences in the net income of those olive tree farmers that develop a traditional growing system as opposed to the rest of cultivations that use the strategies proposed. The results show that the implementation of the here proposed strategic alternatives increase the competitiveness of the traditional cultivation system through the net income of the olive farmer.

KEY-WORDS: Competitiveness - Olive cultivation systems - Olive oil - Profitability - Strategies - Traditional olive cultivation.

\section{INTRODUCCIÓN}

La evolución que viene experimentando la economía mundial, donde la globalización se ha configurado como uno de los rasgos que la caracterizan y definen desde finales del siglo pasado, ha creado el entorno apropiado para el incremento de la competencia entre sus participantes, por lo que el sector agrario, en general, se ha visto involucrado en un necesario proceso de reestructuración (Fuentes, 1999; Etxezarreta, 2006).

Dentro del sector agrario, el sector oleícola asume un elevado y creciente protagonismo a nivel mundial llegando a alcanzar cifras de negocio que oscilan entre los 8.500 y 10.600 millones de euros por ejercicio y obteniéndose producciones medias de aceite de oliva en torno a 3 millones de $t$, de las que 2,2 millones de t corresponden a la Unión Europea y dentro de ésta 1,2 millones de t a España, donde la provincia de Jaén, primera productora a nivel mundial de aceite de oliva, representa el 
40,83\% (COI, 2009). Como sistema de explotación de olivar más extendido en esta provincia se encuentra el cultivo extensivo o tradicional, representando más del $97 \%$ de la superficie de olivar, siendo residuales el resto de modos de explotación, intensivo y superintensivo.

Los últimos cambios acontecidos en el sector, derivados del proceso de reforma experimentado por la Política Agrícola Comunitaria (PAC) y la Organización Común de Mercado (OCM) del olivar, junto con la incorporación de nuevos países en la explotación de este cultivo, ha originado que la competitividad de las explotaciones tradicionales se esté viendo comprometida, al quedar cada vez más expuestas a las fuerzas del libre mercado. Estos hechos se agravan aun más si tenemos en cuenta que las nuevas plantaciones están siendo explotadas de forma intensiva y superintensiva, con menores costes totales de producción que las cultivadas de forma tradicional (Vilar et al., 2010a), con lo que se deja al cultivo tradicional en una posición de inferior competitividad.

Por tanto, para los olivicultores tradicionales, la competitividad se establece en el ámbito de los costes de producción, por lo que para abaratarlos es preciso que reorienten sus modos de explotación implementando estrategias que aporten competitividad y aseguren su supervivencia.

La tesis que defendemos en este trabajo es la siguiente: puesto que la literatura existente respalda que el olivar tradicional está viendo reducida su competitividad, si éste adopta algunas de las estrategias que aquí se presentan (estrategias de modernización de la explotación y cooperación) su competitividad se verá mejorada en términos de renta neta del olivicultor. Esta tesis fue experimentada a través de una prueba piloto (Vilar et al., 2010b) y ello nos ha llevado a plantear este estudio empírico para ratificar dichos resultados y permitir hacerlos extensibles al resto del sector olivarero tradicional que presente condiciones similares a las analizadas. Para alcanzar nuestro objetivo se analizaron datos obtenidos a través de la complementación in situ de cuestionarios a una muestra de 40 explotaciones olivareras tradicionales, 10 por cada una de las alternativas estratégicas formuladas.

El trabajo se ha estructurado como sigue: tras esta introducción, dedicamos un segundo epígrafe a presentar el marco teórico que sustenta la investigación, centrado en un análisis de las singularidades de las estrategias propuestas. En el tercero, se explica detalladamente el método de investigación llevado a cabo. En el cuarto epígrafe se ofrecen los resultados del trabajo y, finalmente, se acaba con unas conclusiones.

\section{MARCO TEÓRICO}

Para hacer frente a la continua caída de competitividad de las explotaciones tradicionales las distintas alternativas que se formulan en este trabajo se encuadran en la estrategia competitiva de cos- tes, y se apoyan en la teoría de sistemas (La teoría de sistemas parte de la idea básica de que un sistema es un conjunto de elementos que interaccionan entre ellos de manera dinámica y que están organizados en función de un objetivo o finalidad, formando un todo unitario, distinto a las partes y más que la suma de las mismas. Estas partes están interrelacionadas e influyen sobre el funcionamiento del sistema) (Speddimg, 1982; Bonneval, 1993; Kast y Rosenzweig, 1987; Cuervo, 1994; León-Velarde et al., 2002) así como en numerosos trabajos de investigación relacionados con la competitividad de las empresas (Kyritsis et al., 1994; Ramos, 1995; Fernández, 2003).

Igualmente, se ha tomado como referencia la legislación y normativa vigente referente a la mejora de estructuras agrarias, tales como, la Ley 19/1995, de 4 de julio, de Modernización de las Explotaciones Agrarias y el Real Decreto 613/2001, de 8 de junio, para la mejora y modernización de las estructuras de producción de las explotaciones agrarias, cuyo objetivo fundamental es corregir los desequilibrios y las deficiencias estructurales que condicionan la competitividad de las explotaciones agrarias, para lo que contemplan ayudas para inversiones que impliquen la adaptación de las explotaciones con vistas a reducir los costes de producción, ahorrar energía o agua, o la incorporación de nuevas tecnologías, incluidas las de informatización y telemática, entre otras.

En cuanto a la teoría de sistemas esta nos permite considerar a las explotaciones olivareras tradicionales como unidades incluidas dentro del sistema agrario, integradas en el sector del olivar con el que interactúan constantemente, al que contribuyen y del que perciben influencia, resaltando que de la interacción y cooperación de este tipo de explotaciones se obtienen efectos sinérgicos, con los consiguientes beneficios individuales y colectivos que ello conlleva (León-Velarde et al., 2002). Son muchos los autores que analizan los efectos positivos de la cooperación, así como de la introducción de innovaciones en el desempeño de la actividad (Urra, 1999; Shapiro, 2005; Rialp, 1996; Miranda, 1992).

En este sentido, las alternativas estratégicas que proponemos se integran en un proceso de innovación, vía modernización de las explotaciones tradicionales, y hacia la búsqueda de distintas formas de cooperación entre empresas del sector del olivar tradicional, que permitirán al olivicultor tradicional llevar a cabo su actividad en explotaciones rentables, evitando su abandono y garantizando de este modo su supervivencia.

La primera de las estrategias es la de modernización de la explotación, consistente en la evolución, adecuación y avance de las explotaciones tradicionales aplicando determinadas mejoras o modificaciones que impliquen cambios en las pautas de comportamiento que el olivicultor experimenta en el desempeño de su actividad, manteniendo o incrementando su efectividad. Se trata de modernizar el sistema de cultivo, adaptándolo a las 
nuevas formas de mecanización, lo que redundará en una reducción del coste de mano de obra, necesaria para llevar a cabo las tareas propias del cultivo del olivo, y en especial la recolección.

La segunda de las estrategias sería la de cooperación, orientada hacia la búsqueda del efecto crecimiento o concentración, concretamente, hacia un cultivo compartido o un cultivo asistido, que implica, partiendo de una primera adecuación de la explotación, poder realizar su actividad de forma coordinada o agrupada con otras empresas del sector del olivar tradicional, obteniendo rentabilidades superiores. En este sentido, la mayor parte de los olivareros tradicionales no desempeñan la actividad de explotación como ocupación principal (en España superan el $75 \%$, elevándose al $80 \%$ en Andalucía (MARM, 2009), mientras motivado en gran medida por la excesiva atomización (más del $80 \%$ de las explotaciones en España no supera las 15 hectáreas (EUROSTAT, 2009) y dispersión de los olivares, lo que impide el desarrollo de la actividad de manera profesionalizada y competitiva. En consecuencia, esta estrategia se configura como una alternativa ante el posible abandono de las explotaciones.

El cultivo compartido como mecanismo de integración horizontal permite el intercambio de conocimiento entre entidades cooperantes produciéndose efectos sinérgicos y gozando de ventajas de flexibilidad y de mejora de posicionamiento en el mercado. Este tipo de cultivo sería la evolución totalmente contraria a la que se produce en el reparto o asignación de herencias (segregación). De hecho este sistema está siendo utilizado y es especialmente indicado para trabajar entre explotaciones contiguas y cercanas, así como entre propietarios de partes alícuotas de fincas. Si dicho crecimiento se produce de manera concéntrica o colindante, reduciendo la dispersión, se eliminan tiempos muertos, periodos in itinere, etc. para cada uno de los procesos o tareas necesarias en la explotación tradicional de olivar, optimizando dicha actividad en mayor medida.

Por su parte, el cultivo asistido consiste en el trabajo integral de las distintas explotaciones olivareras por una empresa de servicios profesionales. Su nivel de eficacia y eficiencia, es tanto más alto, cuanto mayor es el número de fincas y superior el índice de concentración entre la mismas.

De este modo, el componente activo o poseedor de recursos, optimiza el uso de los mismos, a la vez que reduce los costes de amortización, mientras que los titulares pasivos o prestatarios de servicios, reducen el riesgo, o rango de la inversión, a la vez que facilita los intercambios, relaciones, gestión y administración de la explotación, proporciona mayor disponibilidad de personal, y mejor posición ante suministradores y proveedores (optimiza la posición negociadora), con la consiguiente reducción de costes.

Los planteamientos aquí expuestos fueron ensayados a través de una prueba piloto (Vilar et al., $2010 b)$, que corroboró en una primera instancia nuestro marco teórico, en cuanto a mejora de la renta neta y competitividad de las explotaciones tradicionales que aplicaban las medidas propuestas (Tabla 1). Ello nos ha llevado a plantear este estudio empírico para ratificar dichos resultados y permitir hacerlos extensibles al resto del sector olivarero tradicional que presente condiciones similares a las analizadas.

\section{DISEÑO DE LA INVESTIGACIÓN}

\subsection{Hipótesis de Investigación}

Considerando el fin de la investigación, ya enunciado en la introducción y marco teórico, y tomando como referencia las diversas estrategias de modernización de la explotación y cooperación que se proponen en el artículo, nuestras hipótesis de investigación son las siguientes:

H1: Existe incidencia de la modernización de la explotación, vía evolución, avance y adecuación, sobre la competitividad de las explotaciones de olivar tradicional en función de la renta neta del olivicultor, en adelante, competitividad de la renta neta.

H2: Existe incidencia del cultivo compartido sobre la competitividad de la renta neta.

H3: Existe incidencia del cultivo asistido o subcontratado sobre la competitividad de la renta neta.

\subsection{Selección de la Muestra}

Para llevar a cabo el estudio elegimos una población de explotaciones con límite finito, en este caso 40 , todas ellas distribuidas en la provincia de Jaén (España), por la importancia que tiene la misma en términos de producción a nivel mundial.

Para evitar sesgos y posibles errores, todas y cada una de las explotaciones, tienen características similares como son:

Tabla 1

Comparativa de la renta neta del olivicultor y costes de recolección en función de la alternativa estratégica propuesta extraídos de la prueba piloto

\begin{tabular}{lcc}
\hline \multicolumn{1}{c}{ Alternativa estratégica propuesta } & Renta neta del olivicultor & Costes de recolección \\
\hline Modernización de la explotación & $2.879,57 €$ & $667,08 €$ \\
Tradicional compartido & $3.423,81 €$ & $495,12 €$ \\
Tradicional asistido & $3.875,97 €$ & $472,57 €$ \\
\hline
\end{tabular}

Fuente: Elaboración propia a partir Vilar et al., 2010b 
- Las explotaciones analizadas contienen el mismo número de olivos por hectárea, entre 97 y 100 olivos.

- El 92 por 100 de las fincas no alcanza las 15 hectáreas.

- Todas las fincas son de la variedad de olivo picual y poseen entre 2 y 4 pies.

- Se encuentran a una altitud de entre 550 y 700 metros sobre el nivel del mar, de orografía similar, de secano y ubicadas en la misma comarca.

- Ninguna de las explotaciones dedica parte de su producción a aceituna de mesa, por lo que su destino es exclusivo para almazara.

- Para el 50 por 100 de sus titulares, la explotación de ese cultivo, no constituye su actividad principal.

- La subvención de la Unión Europea representa entre el 25 y 40 por 100 del total de los ingresos percibidos por la explotación de esta actividad.

En todas las categorías de olivar analizadas la recolección fue realizada en enero, en días con características climatológicas similares, e igualdad de recursos técnicos, humanos, etc. todo ello para la campaña 2008/09. Además todas las explotaciones son proveedoras de la misma almazara, estableciéndose como precio de venta para el cálculo de ingresos la media para dicha almazara durante la anterior campaña, como consecuencia de no haberse realizado aún la venta del aceite en la campaña actual.

\subsection{Procedimiento}

Con el propósito de obtener los datos para la comprobación de las hipótesis, se siguió como mé- todo de trabajo la investigación por encuesta. Más concretamente se utilizó una tabla de trabajo (Tabla 2), dirigida a los propietarios de las explotaciones, por considerar que éste es el método más fiable con que cuentan los investigadores sociales para describir y analizar una población a la cual no podemos observar de modo directo por su amplitud.

Las partidas incluidas en ella están justificadas a partir de su utilización por numerosos autores en estudios de costes locales, provinciales, nacionales e internacionales como Alonso y Serrano (1991), Lovera (1995), Cámara (1997), Civantos (1997), Cámara y Sánchez (1998), Sánchez (2002), Guerrero (2003), Vilar (2006), Navarro (2007) y Barranco et al. (2008).

En dicha tabla de trabajo se incluyen los costes necesarios para desarrollar la explotación del olivo, a partir de las distintas tareas requeridas para el desempeño de la actividad olivarera hasta la obtención de aceite, así como los ingresos relativos a dicha actividad. La partida de renta neta o beneficio neto es la que se ha utilizado para comparar desde el punto de vista económico las distintas estrategias de competitividad propuestas. Así su utilización viene justificada por considerarse una partida indispensable para determinar la rentabilidad de las explotaciones. Así Gitman (1997) dice que rentabilidad es la relación entre ingresos y costos generados por el uso de los activos de la empresa en actividades productivas. La rentabilidad de una empresa puede ser evaluada en referencia a las ventas, a los activos, al capital o al valor accionario. Por otra parte, Aguirre et al. (1997) consideran la rentabilidad como un objetivo económico a corto plazo que las empresas deben alcanzar, relaciona-

Tabla 2

Modelo de tabla para el análisis económico de actividad para las explotaciones de olivar

\begin{tabular}{|c|c|c|}
\hline Producción por hectárea & $\begin{array}{l}\text { Gastos } \\
\text { (euros) }\end{array}$ & $\begin{array}{c}\text { Ingresos } \\
\text { (euros) }\end{array}$ \\
\hline \multicolumn{3}{|l|}{$\mathrm{Kg} /$ olivo } \\
\hline \multicolumn{3}{|l|}{ Ingresos } \\
\hline \multicolumn{3}{|l|}{ Subvención a la producción } \\
\hline \multicolumn{3}{|l|}{ Poda y eliminación de leña (bienal) } \\
\hline \multicolumn{3}{|l|}{ Arijas (arado, rastreado, etc.) } \\
\hline \multicolumn{3}{|l|}{ Abonado / estercolado } \\
\hline \multicolumn{3}{|l|}{ Tratamientos (repilo, prays y mosca) } \\
\hline \multicolumn{3}{|l|}{ Despestugo / desvareto } \\
\hline \multicolumn{3}{|l|}{ Recolección } \\
\hline \multicolumn{3}{|l|}{ Transporte de tajo a almazara } \\
\hline \multicolumn{3}{|l|}{ Extracción (euros / Kg.) } \\
\hline \multicolumn{3}{|l|}{ Total } \\
\hline \multicolumn{3}{|l|}{ Renta Neta } \\
\hline \multicolumn{3}{|l|}{ Valor inmovilizado de mercado (euros) } \\
\hline Margen neto de Explotación (\%) & & \\
\hline
\end{tabular}


do con la obtención de un beneficio necesario para el buen desarrollo de la empresa.

Las características básicas de la investigación nos vienen determinadas por la siguiente ficha técnica (Tabla 3).

Tabla 3

Ficha técnica del estudio de investigación

\begin{tabular}{l}
\hline Población \\
\hline Universo: 131.000 explotaciones olivareras \\
tradicionales de la provincia de Jaén. \\
Ámbito geográfico: Provincial. \\
Tamaño de la muestra: 40 explotaciones. \\
Intervalo temporal de trabajo de campo: \\
diciembre 2008-enero 2009. \\
\hline Muestreo \\
\hline Censo/diseño muestral: Muestreo aleatorio simple. \\
Error muestral: 0,1549 \\
Nivel de confianza: $95,5 \% \mathrm{Z}=1,96 \mathrm{p}=\mathrm{q}=0,5$
\end{tabular}

Fuente: Elaboración propia.

En lo que respecta al error muestral, nos sirve de orientación en cuanto a ratificación en la validez de la muestra objeto de estudio, por muestreo aleatorio simple, siendo por tanto los resultados válidos para su extrapolación a la totalidad de la población que presente condiciones similares a las analizadas.

Con objeto de llevar a cabo el tratamiento estadístico, se procede a realizar un análisis de la varianza (ANOVA) que sirve para comparar varios grupos en una variable cuantitativa (Luque, 2000).

A la variable que define los grupos que se desea comparar se le llama independiente o factor, que en este caso son los cuatro distintos modos de explotación de olivar: sistema tradicional; cultivo tradicional modernizado; cultivo compartido y por último, explotación asistida. La variable cuantitativa en la que deseamos comparar los grupos es la variable dependiente que en nuestro caso, es la renta neta por hectárea.

La hipótesis estadística que se quiere contrastar en la ANOVA consiste en determinar si las medias poblacionales de la variable dependiente, en cada uno de los valores de la variable del factor, son iguales.

Con el fin de dotar nuestro análisis de un mayor rigor estadístico en cuanto a resultado e interpretación, se realizaron las pruebas de Brown-Forsythe y Welch, dichas pruebas son robustas, en el sentido de que son alternativas al ANOVA en caso de que no se verifique la hipótesis de igualdad de varianzas. Así, estas pruebas permitirán comprobar que realmente los resultados son los mismos en caso de que no se confirme la hipótesis de homocedasticidad.

En caso de rechazar la hipótesis nula sabremos que, al menos, hay diferencias entre al menos dos de las medias, es decir, se pondrá de manifiesto, que mediante la aplicación de los distintos siste- mas de cultivo, la renta neta percibida por el olivicultor es diferente, por tanto, resultaría adecuado llevar a cabo alguna modificación en el sistema de explotación, pues afectará a la competitividad, y por tanto, a los posibles incrementos de renta.

En caso de que las pruebas estadísticas confirmen la existencia de diferencias significativas entre las medias de la variable dependiente (renta neta por hectárea), según el tipo de cultivo o explotación- se realizarán comparaciones dos a dos para saber qué medias son estadísticamente diferentes (se desconoce entre qué medias hay diferencia, para tener esta información hay que realizar un análisis post hoc o a posteriori en el que se comparen las medias dos a dos).

Para ello se han considerado dos pruebas, la prueba de Tukey, que asume varianzas iguales, y la prueba de Games-Howell, que es similar a la anterior pero que no asume varianzas iguales.

Por último, se realizará un análisis de homogeneidad de grupos con el objetivo de obtener posibles grupos de explotaciones que en función de su puntuación, sean homogéneos con respecto al grupo que forman, y heterogéneos con los componentes del resto de los elementos.

\section{RESULTADOS}

A continuación se procede a comprobar la incidencia que sobre la renta neta del olivicultor tradicional, tienen las estrategias de modernización y cooperación propuestas, y más concretamente, el cultivo modernizado de olivar tradicional, el modo de explotación compartida y el sistema de cultivo asistido.

En la tabla 4, se muestra un resumen estadístico descriptivo de la variable renta neta, el número de casos por tipo de cultivo, la media muestral, la desviación típica muestral, el error típico (la desviación típica dividida por la raíz cuadrada del número total de casos) y el intervalo de confianza para la media. Aparece una medida para cada muestra (definida por cada tipo de cultivo), y otra para el global.

Como se observa en la tabla ANOVA (Tabla 5) el p-valor es cero, por lo que a cualquier nivel de significación que queramos tomar $(0.05,0.01,0.1)$ se rechaza la hipótesis nula y por lo tanto hay diferencias significativas entre los valores medios de la renta neta por hectárea para cada tipo de cultivo.

Por tanto, corroboramos estadísticamente que existe incidencia de la modernización y de los acuerdos de cooperación sobre la competitividad de las explotaciones de olivar tradicional en función de la renta neta del olivicultor.

Además, los resultados de las pruebas de Brown-Forsythe y Welch (Tabla 6) vienen a corroborar las conclusiones hasta ahora obtenidas. Se obtiene un p-valor en ambos casos de cero, por lo que a cualquier nivel de significación que queramos tomar $(0.05,0.01,0.1)$ se rechaza la hipótesis nula. 
Tabla 4

Resumen estadístico descriptivo de la variable "renta neta"

\begin{tabular}{|c|c|c|c|c|c|c|c|c|}
\hline & \multirow{2}{*}{$\mathbf{N}$} & \multirow{2}{*}{ Media } & \multirow{2}{*}{$\begin{array}{l}\text { Desviación } \\
\text { Típica }\end{array}$} & \multirow{2}{*}{$\begin{array}{l}\text { Error } \\
\text { Típico }\end{array}$} & \multicolumn{2}{|c|}{$\begin{array}{l}\text { Intervalo de confianza } \\
\text { para la media al } 95 \%\end{array}$} & \multirow{2}{*}{ Mínimo } & \multirow{2}{*}{ Máximc } \\
\hline & & & & & $\begin{array}{l}\text { Límite } \\
\text { inferior }\end{array}$ & $\begin{array}{l}\text { Límite } \\
\text { superior }\end{array}$ & & \\
\hline Tradicional & 10 & 1738,90 & 145,82 & 46,11 & 1634,59 & 1843,21 & 1460,00 & 1912,00 \\
\hline $\begin{array}{l}\text { Tradicional } \\
\text { modernizado }\end{array}$ & 10 & 2764,80 & 114,05 & 36,07 & 2683,21 & 2846,39 & 2586,00 & 2900,00 \\
\hline $\begin{array}{l}\text { Cultivo } \\
\text { compartido }\end{array}$ & 10 & 3374,20 & 224,82 & 71,09 & 3213,37 & 3535,03 & 3120,00 & 3680,00 \\
\hline $\begin{array}{l}\text { Cultivo } \\
\text { asistido }\end{array}$ & 10 & 3770,00 & 83,13 & 26,29 & 3710,53 & 3829,47 & 3650,00 & 3900,00 \\
\hline Total & 40 & 2911,98 & 789,38 & 124,81 & 2659,52 & 3164,43 & 1460,00 & 3900,00 \\
\hline
\end{tabular}

Fuente: Elaboración propia.

Tabla 5

Prueba ANOVA para la variable "diferencia de renta neta"

\begin{tabular}{lccccc}
\hline & Suma de cuadrados & GI & Media cuadrática & f & Sig. \\
\hline Inter-grupos & 23476242,88 & 3 & 7825414,29 & 341,25 & 0,00 \\
\hline Intra-grupos & 825530,10 & 36 & 22931,39 & & \\
\hline Total & 24301772,98 & 39 & & & \\
\hline
\end{tabular}

Fuente: Elaboración propia.

Tabla 6

Prueba robustez de igualdad de medias para la variable "diferencia de renta neta"

\begin{tabular}{lcccc}
\hline & Estadístico (a) & Gl1 & GI2 & Sig. \\
\hline Welch & 503,73 & 3 & 19,12 & 0,00 \\
\hline Brown-Forsythe & 341,25 & 3 & 23,49 & 0,00 \\
\hline
\end{tabular}

Fuente: Elaboración propia.

Así, para saber qué parejas de medias son estadísticamente diferentes en la Tabla 7 , se presentan, aplicando la prueba de Tukey y la de Games Hewell, todas las combinaciones dos a dos entre los niveles de la variable Tipo de Cultivo, las diferencias entre los valores medios de la variable renta neta por hectárea para cada uno de los grupos, y el p-valor asociado a cada una de las pruebas realizadas.

Como vemos en la tabla, ambas pruebas nos indican que hay diferencias significativas entre todos los pares de medias (están todos marcados con un *), al nivel de significación del 5 por 100 . Por tanto, a tenor de los datos, se vuelven a corroborar los resultados obtenidos, quedando contrastados con las anteriores pruebas.

Por otra parte, el análisis de homogeneidad de grupos (Tabla 8), que nos permite tener una clasificación de los grupos en función de lo parecidas que sean sus medias, nos muestra que hay cuatro grupos, puesto que hay diferencias entre los cuatro niveles del factor. También se puede observar que el valor medio de la renta media por hectárea con el cultivo tradicional es menor que la obtenida con el tradicional modernizado, que es a su vez menor que la obtenida con el cultivo compartido, que es menor que la obtenida con el cultivo asistido.

Gráficamente (Figura 1), podemos representar las medias de la variable renta neta por hectárea frente a la variable modo de cultivo, que nos ilustra la conclusión anterior del orden de las medias.

A la vista de los resultados estadísticos obtenidos en este estudio se puede afirmar que la aplicación de las medidas de modernización de la explotación y la cooperación en las explotaciones de olivar tradicional eleva la competitividad y por ende la renta neta del olivicultor, por tanto, se cumplen nuestras hipótesis.

\section{CONCLUSIONES}

Mediante la aplicación de las estrategias propuestas de modernización y cooperación se pretende mejorar la competitividad del olivar tradicional que adolece de una excesiva atomización, dispersión y elevados costes de explotación, proporcionando incrementos en la renta neta del olivicultor.

El trabajo corrobora todas nuestras hipótesis de partida y podemos concluir que: 
Tabla 7

Prueba post hoc de comparaciones múltiples para la variable "diferencia de renta neta"

\begin{tabular}{|c|c|c|c|c|c|c|c|}
\hline & \multirow{2}{*}{$\begin{array}{l}\text { (I) Tipo } \\
\text { Cultivo }\end{array}$} & \multirow{2}{*}{$\begin{array}{l}\text { (J) Tipo } \\
\text { Cultivo }\end{array}$} & \multirow{2}{*}{$\begin{array}{l}\text { Diferencia de } \\
\text { medias (I-J) }\end{array}$} & \multirow{2}{*}{$\begin{array}{l}\text { Error } \\
\text { típico }\end{array}$} & \multirow[b]{2}{*}{ Sig. } & \multicolumn{2}{|c|}{ Intervalo de confianza al $95 \%$} \\
\hline & & & & & & $\begin{array}{l}\text { Límite } \\
\text { superior }\end{array}$ & $\begin{array}{l}\text { Límite } \\
\text { inferior }\end{array}$ \\
\hline \multirow[t]{12}{*}{ HSD de Tukey } & \multirow{3}{*}{ Tradicional } & $\begin{array}{l}\text { Tradicional } \\
\text { modernizado }\end{array}$ & $-1025,90\left(^{\star}\right)$ & 67,72 & 0,00 & $-1208,29$ & $-843,51$ \\
\hline & & $\begin{array}{l}\text { Cultivo } \\
\text { compartido }\end{array}$ & $-1635,30\left(^{*}\right)$ & 67,72 & 0,00 & $-1817,69$ & $-1452,91$ \\
\hline & & Cultivo asistido & $-2031,10\left(^{\star}\right)$ & 67,72 & 0,00 & $-2213,49$ & $-1848,71$ \\
\hline & \multirow{3}{*}{$\begin{array}{l}\text { Tradicional } \\
\text { modernizado }\end{array}$} & Tradicional & $1025,90\left(^{*}\right)$ & 67,72 & 0,00 & 843,51 & 1208,29 \\
\hline & & $\begin{array}{l}\text { Cultivo } \\
\text { compartido }\end{array}$ & $-609,40\left(^{\star}\right)$ & 67,72 & 0,00 & $-791,79$ & $-427,01$ \\
\hline & & Cultivo asistido & $-1005,20\left(^{*}\right)$ & 67,72 & 0,00 & $-1187,59$ & $-822,81$ \\
\hline & \multirow{3}{*}{$\begin{array}{l}\text { Cultivo } \\
\text { compartido }\end{array}$} & Tradicional & $1635,30\left(^{*}\right)$ & 67,72 & 0,00 & 1452,91 & 1817,69 \\
\hline & & $\begin{array}{l}\text { Tradicional } \\
\text { modernizado }\end{array}$ & $609,40\left(^{*}\right)$ & 67,72 & 0,00 & 427,01 & 791,79 \\
\hline & & Cultivo asistido & $-395,80\left(^{*}\right)$ & 67,72 & 0,00 & $-578,19$ & $-213,41$ \\
\hline & \multirow{3}{*}{$\begin{array}{l}\text { Cultivo } \\
\text { asistido }\end{array}$} & Tradicional & $2031,10\left(^{\star}\right)$ & 67,72 & 0,00 & 1848,71 & 2213,49 \\
\hline & & $\begin{array}{l}\text { Tradicional } \\
\text { modernizado }\end{array}$ & $1005,20\left(^{*}\right)$ & 67,72 & 0,00 & 822,81 & 1187,59 \\
\hline & & $\begin{array}{l}\text { Cultivo } \\
\text { compartido }\end{array}$ & $395,80\left(^{*}\right)$ & 67,72 & 0,00 & 213,41 & 578,19 \\
\hline \multirow[t]{12}{*}{ Games-Howell } & \multirow{3}{*}{ Tradicional } & $\begin{array}{l}\text { Tradicional } \\
\text { modernizado }\end{array}$ & $-1025,90\left(^{*}\right)$ & 58,54 & 0,00 & $-1192,29$ & $-859,51$ \\
\hline & & $\begin{array}{l}\text { Cultivo } \\
\text { compartido }\end{array}$ & $-1635,30\left(^{\star}\right)$ & 84,74 & 0,00 & $-1878,72$ & $-1391,88$ \\
\hline & & Cultivo asistido & $-2031,10\left(^{*}\right)$ & 53,08 & 0,00 & $-2184,98$ & $-1877,22$ \\
\hline & \multirow{3}{*}{$\begin{array}{l}\text { Tradicional } \\
\text { modernizado }\end{array}$} & Tradicional & $1025,90\left(^{*}\right)$ & 58,54 & 0,00 & 859,51 & 1192,29 \\
\hline & & $\begin{array}{l}\text { Cultivo } \\
\text { compartido }\end{array}$ & $-609,40\left(^{\star}\right)$ & 79,72 & 0,00 & $-842,56$ & $-376,24$ \\
\hline & & Cultivo asistido & $-1005,20\left(^{*}\right)$ & 44,63 & 0,00 & $-1132,50$ & $-877,90$ \\
\hline & \multirow{3}{*}{$\begin{array}{l}\text { Cultivo } \\
\text { compartido }\end{array}$} & Tradicional & $1635,30\left(^{*}\right)$ & 84,74 & 0,00 & 1391,88 & 1878,73 \\
\hline & & $\begin{array}{l}\text { Tradicional } \\
\text { modernizado }\end{array}$ & $609,40\left(^{*}\right)$ & 79,72 & 0,00 & 376,24 & 842,56 \\
\hline & & Cultivo asistido & $-395,80\left(^{*}\right)$ & 75,80 & 0,001 & $-622,57$ & $-169,03$ \\
\hline & \multirow{3}{*}{$\begin{array}{l}\text { Cultivo } \\
\text { asistido }\end{array}$} & Tradicional & $2031,10\left(^{*}\right)$ & 53,08 & 0,00 & 1877,22 & 2184,98 \\
\hline & & $\begin{array}{l}\text { Tradicional } \\
\text { modernizado }\end{array}$ & $1005,20\left(^{\star}\right)$ & 44,63 & 0,00 & 877,90 & 1132,50 \\
\hline & & $\begin{array}{l}\text { Cultivo } \\
\text { compartido }\end{array}$ & $395,80\left(^{*}\right)$ & 75,80 & 0,001 & 169,03 & 622,57 \\
\hline
\end{tabular}

* La diferencia de medias es significativa al nivel 0,05. Fuente: Elaboración propia.

- Existe incidencia de la modernización de las explotaciones de olivar tradicional, sobre la competitividad de las explotaciones de olivar tradicional en la renta neta del olivicultor.

- Existe incidencia del cultivo compartido de olivar tradicional sobre la competitividad de la renta neta del olivicultor.
- Existe incidencia del cultivo asistido o subcontratado de olivar tradicional sobre la competitividad de la renta neta del olivicultor.

En este sentido, los datos estadísticos ponen de manifiesto que de forma gradual, se produce un incremento significativo de la renta neta del cultivo 
Tabla 8

Tabla de subconjuntos homogéneos para la variable "diferencia de renta neta"

\begin{tabular}{|c|c|c|c|c|c|c|}
\hline & \multirow[t]{2}{*}{ Tipo cultivo } & \multirow{2}{*}{$\begin{array}{l}\mathrm{n} \\
1\end{array}$} & \multicolumn{4}{|c|}{ Subconjunto para alfa $=0,05$} \\
\hline & & & 2 & 3 & 4 & 1 \\
\hline \multirow[t]{5}{*}{ HSD de Tukey(a) } & Tradicional & 10 & 1738,90 & & & \\
\hline & Tradicional modernizado & 10 & & 2764,80 & & \\
\hline & Cultivo compartido & 10 & & & 3374,20 & \\
\hline & Cultivo asistido & 10 & & & & 3770,00 \\
\hline & Sig. & & 1.00 & 1.00 & 1.00 & 1.00 \\
\hline
\end{tabular}

Se muestran las medias para los grupos en los subconjuntos homogéneos. (a): Usa el tamaño muestral de la media armónica = 10. Fuente: Elaboración propia.

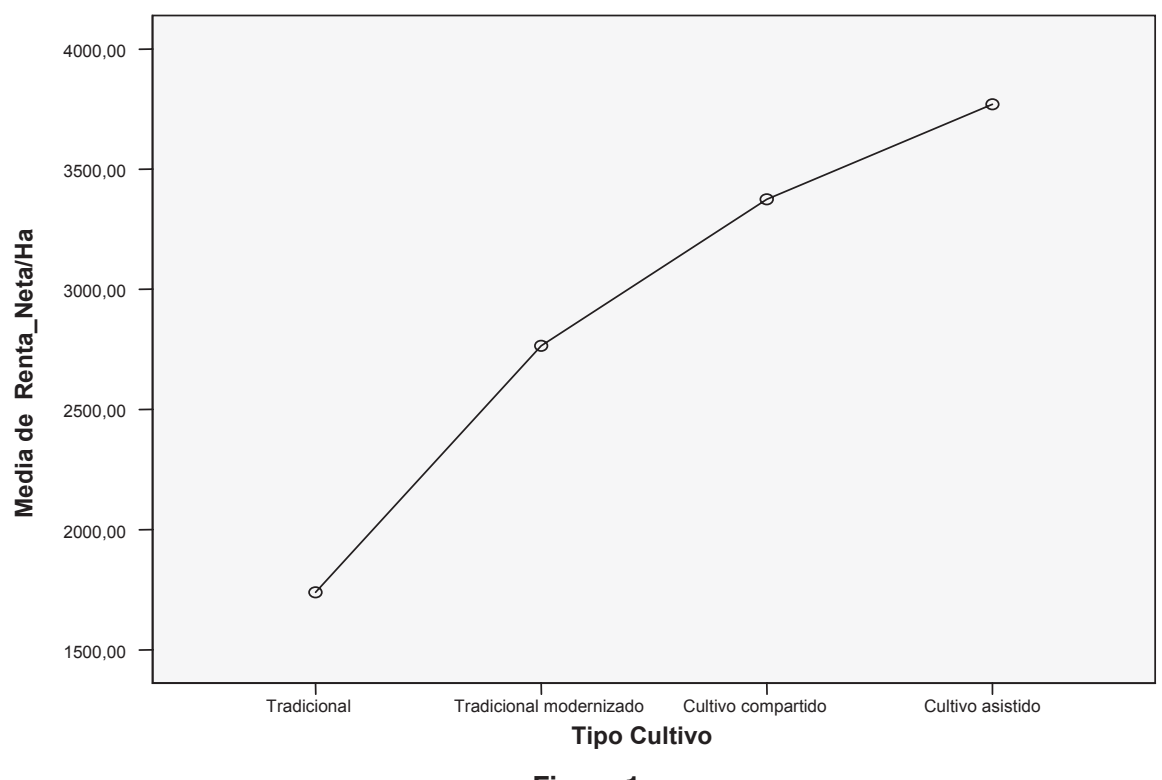

Figura 1

Relación de medias de renta neta / hectárea por sistema de cultivo. Fuente: Elaboración propia.

tradicional, al aplicar medidas de modernización, y que de la misma manera que por el efecto concentración y cooperación en el cultivo compartido y en la explotación asistida se vuelve a producir una mejora competitiva, y por tanto un nuevo incremento de renta neta, siendo superior al resto, sin discusión alguna, para la explotación asistida.

\section{REFERENCIAS}

Aguirre J. Prieto M. Escamilla J. 1997. Contabilidad de costos, gestión y control presupuestario, control de gestión, la función del controller. Tomo II. Cultural de Ediciones, S.A. Madrid.

Alonso R, Serrano A. 1991. Los costes en los procesos de producción agraria. Mundi-Prensa. Madrid.

Barasona J. 1994. Mecanización de la recolección de la aceituna. Agricultura 746, 13-19.

Barranco D, Fernández-Escobar R, Rallo L. 2008. El cultivo del olivo. Mundi-Prensa. Madrid.

Bonneval L. 1993. Systemes agraries. Systemes de production. Vocabulaire. Ediciones INRA. Paris.

Cámara M. 1997. Gestión medioambiental y contabilidad. Una aplicación al sector del aceite de oliva. Di- putación Provincial de Jaén. Instituto de Estudios Giennenses, Jaén.

Cámara M, Sánchez S. 1998. Los costes de los cultivos de olivar y la política medioambiental de las almazaras jiennenses. Primeras Jornadas Mediterráneas de Olivar Ecológico. Ecoliva'97. Puente de Génave, noviembre, págs. 221-245.

Civantos L. 1997. La olivicultura en el mundo y en España. El Cultivo del olivo. Junta de Andalucía, Madrid.

Consejo Oleícola Internacional (COI). 2009. Producción, importación, exportación y consumo mundial de aceite de oliva 1991-2008/09. www.internationaloliveoil. org.

Cuervo A. 1994. Introducción a la Administración de Empresas. Cívitas. Madrid.

Etxezarreta M. 2006. Tendencias de evolución de la agricultura al principio del siglo XXI, en Etxezarreta M. (dir) La agricultura española en la era de la globalización. Ministerio de Agricultura, Pesca y Alimentación. Madrid, págs. 13-25.

Fernández MA. 2003. Viabilidad y competitividad de los sistemas hortofrutícolas mediterráneos según su grado de intensificación. Tesis doctoral. Universidad de Valencia. Escuela Técnica Superior de Ingenieros Agrónomos. Departamento de Producción Vegetal.

Fuentes FJ. 1999. Presente y Futuro de las empresas agrarias andaluzas de cereal y olivar. Un análisis em- 
pírico. Publicaciones de la Universidad de Córdoba y Obra Social y Cultural Cajasur. Córdoba.

Gitman L. 1997. Fundamentos de administración financiera. Editorial OUP Harla México, S.A. México.

Guerrero A. 2003. Nueva olivicultura. Mundi-Prensa. Madrid.

Humanes J, Pastor M, Vega V, Castro J. 1998. Diseño y manejo de plantaciones de oliva. Junta de Andalucía. Consejería de Agricultura y Pesca. Sevilla.

Kast FE, Rosenzweig JE. 1987. Organization and management: a systems and contingency approach. MCcGraw-Hill. New York.

Kyritsis S, Soldatos PG, Dent B, Mcgregor MJ. 1994. Biomass as an alternative land use for southern European agriculture. Rural and farming systems analysis: european perspectives (CAB International, Wallingford-UK), 159-166.

León-Velarde C, Reinoso J, Valdivia R. 2002. La investigación de sistemas agropecuarios y su relación en la cadena producción-consumo: evolución metodológica y experiencias en el Altiplano. Disponible en www. rimisp.cl/publicaciones/electrónicas/.

Lovera C. 1995. La modernización agraria ante el reto de la competitividad, en AA.VV. Hacia un nuevo sistema rural. Ed. MAPA- Serie Estudios, págs. 329-344.

Luque T. 2000. Técnicas de análisis de datos en investigación de mercados. Ediciones Pirámide (Grupo Anaya S.A.). Madrid.

Márquez L. 1992. La reducción de los costes por el uso racional de la maquinaria. Adaptación de la maquinaria para nuevos cultivos, en AA.VV. Competitividad de la agricultura española ante el Mercado Único. Tierras de cultivo abandonadas. Agrícola Española S.A. Madrid, págs. 21-139.

Ministerio de Agricultura y Medio Rural y Marino (MARM). 2009. Anuario de estadística. Disponible en http:// www.mapa.es/es/estadística.

Miranda FJ. 1992. Organización de los acuerdos de cooperación interempresarial en España y su entorno. Economía Industrial 285, 7-16.

Navarro C. 2007. Cultivo intensivo de olivar. Plantación, recursos y evolución. Jornadas técnicas el futuro de la olivicultura con suficiencia de recursos "Cultivo intensivo versus superintensivo”. Córdoba, marzo.
Oficina Europea de Estadística (Eurostat). 2009. Europa en cifras. Anuario Eurostat 2009. Eurostat. Statistical Books. Comisión Europea. Luxemburgo.

Ramos E. 1995. El desafío del sector agroalimentario: un reto de competitividad, en Ramos E, Cruz J. (coords.) Hacia un nuevo sistema rural. MAPA-Serie Estudios, págs. 345-380.

Sanchez S. 2002. El cultivo del olivar desde una perspectiva de gestión. Universidad de Jaén. Jaén.

Rialp A. 1996. El papel de los acuerdos de cooperación en los procesos de internacionalización de la empresa española: un análisis empírico. Papeles de Economía Española 66, 248-266.

Shapiro S. 2005. Innovar para ser competitivo. Harvard Deusto Marketing \& Ventas 66, 76-79.

Speddimg CRW. 1982. Sistemas agrarios. Editorial Acribia. Barcelona.

Tamames R, Rueda A. 2005. Estructura económica de España. Alianza Editorial S.A. Madrid.

Tió C. 2007. La reforma de la PAC y sus impacto en el sector olivarero, en Sillero J. (coord.) Congreso de la Cultura del aceite. Instituto de Estudios Giennenses, págs. 323-340.

Urra JA. 1999. Cooperación empresarial: revisión y tendencias. Estudios Financieros 200, 275-396.

Vilar J. 2006. Análisis económico internacional del proceso de elaboración de aceite de oliva. Retos y estrategias de futuro. Westfalia Separator Andalucía, S. L. Úbeda (Jaén).

Vilar J, Velasco MM, Puentes R. 2010a. Estrategias de aplicación para la mejora de la rentabilidad del olivar tradicional. La importancia de la promoción, en Vilar J. et al. (coord.) El Patrimonio Oleícola: Análisis desde la diversidad del conocimiento. Grupo de desarrollo rural de la Sierra Mágina. Jaén, págs. 290-311.

Vilar J, Velasco MM, Puentes R. 2010b. Incidencia del modo de explotación del olivo sobre la renta neta del olivicultor. Estrategias para el cultivo extensivo en el contexto de la posible ausencia de subvenciones. Grasas y Aceites 61, 430-440. 